\title{
Aspectos bioecológicos de Stellifer rastrifer (Perciformes: Sciaenidae) na Baía de Santos, SP
}

\author{
Roberto GIANNINI ${ }^{1}$ \& Alfredo Martins PAIVA FILHO \\ Instituto Oceanográfico da Universidade de São Paulo \\ (Caixa Postal 9075, 01051 São Paulo, SP)
}

\begin{abstract}
- Abstract: As many as 7,260 specimens of the sciaenid Stellifer rastrifer were collected through a monthly beach seine and bottom trawl survey in the complex bay-estuary of Santos and São Vicente, between March 1985 and May 1986. The occurrence, abundance, length frequency, influence of environmental factors, length-weight relationship, growth, recruitment and mortality were analysed.S. rastrifer occurred year-round and was more abundant and frequent in fall at west side of medium and inner stracta. Young of the year was first caught from January to May in deeper water areas and later they were found in shallow areas low with salinity and high temperature. The low catches found in spring may be related to a massive emigration to open sea (summer spawning). S. rastrifer showed higher values for growth coefficient and for total mortality and exploitation rates.
\end{abstract}

- Descriptors: Population structure, Population number, Size distribution, Lengthweight relationship, Growth, Recruitment, Mortality, Environmental effects, Stellifer rastrifer, Sciaenidae, Estuaries, Santos: SP, São Vicente, Brazil.

- Descritores: Estrutura da população, Número da população, Distribuição por tamanho, Relação comprimento/peso, Crescimento, Recrutamento, Mortalidade, Efeitos ambientais, Stelliffer rastrifer, Sciaenidade, Estuários, Santos: SP, Sāo Vicente: SP.

\section{Introdução}

O cangoá Stellifer rastrifer é uma espécie costeira e abundante, sendo encontrada sobre fundos de areia ou lama e em regiōes estuarinas; ocorre da Colômbia ao sul do Brasil e atinge, no máximo, $200 \mathrm{~mm}$ de comprimento total (Chao, 1978; Menezes \& Figueiredo, 1980).

Sua dieta alimentar constituí-se basicamente de pequenos crustáceos e sua captura está relacionada principalmente com a pesca de camarões, sendo a espécie mais abundante na pesca do camarão sete-barbas no Estado de São Paulo(Chao, 1978; Coelhoet al., 1986; Paiva Filho \& Schmiegelow, 1986).

No complexo baía-estuário de Santos e São Vicente foi uma das espécies mais freqüentes e abundantes nos arrastos de fundo na baía e zona costeira adjacente (Paiva Filho et al., 1987).

Neste trabalho foram analisados a ocorrência, a abundância, a distribuição de comprimentos, a influência

(1) Pós-graduando do Departamento de Oceanografia Biológica. Contr. $n^{\circ} 716$ do Inst. oceanogr. da Usp. de fatores ambientais na distribuição da espécie, a relação peso/comprimento, o crescimento, o recrutamento e a mortalidade na Baía de Santos, objetivando o conhecimento dos padrōes de distribuição temporal e espacial, bem como fornecer subsídios para uma explotação racional da espécie na área estudada.

\section{Material e métodos}

A área estudada compreendeu a Baía de Santos, desde sua barra até a região mais interna de praias (Fig. 1); a descrição da área de amostragem bem como uma caracterização da mesma consta de trabalhos recentes (Giannini, 1989; Ribeiro Neto, 1989).

O material analisado foi proveniente de amostras mensais diurnas, realizadas de março de 1985 a maio de 1986, com arrastos de fundo, utilizando-se uma rede de portas de $16,7 \mathrm{~m}$ de largura na tralha inferior e malhas de $20 \mathrm{~mm}$ e $15 \mathrm{~mm}$ (nó a nó) no corpo e sacador respectivamente, e com arrastos de praia, utilizando-se uma rede de calão de $15,0 \mathrm{~m}$ de comprimento, altura de $1,5 \mathrm{~m}$ e malha de $5 \mathrm{~mm}$ (nó a nó). 
A Baía de Santos foi dividida em doze áreas de amostragem, dispostas em três setores para os arrastos de fundo (A, B, C), e em treze áreas de amostragem num único setor para os arrastos de praia (PR); cada setor foi subdividido em lados leste e oeste $(\mathbf{L}, \mathbf{W})$, definindo assim os sub-setores (AL, AW, BL, BW, CL, CW, PRL, PRW) (Fig. 1).

Mensalmente foram efetuadas cerca de oito arrastos de fundo e doze arrastos de praia; para os arrastos de fundo, a unidade de captura por unidade de esforço utilizada foi a da captura total por 5 minutos de arrasto, e para os arrastos de praia, a captura total por $150 \mathrm{~m}$ de distância percorrida no arrasto.

Os arrastos de fundo foram efetuados em todos os setores e sub-setores e em estratos de profundidade distintos (estrato 1, de profundidade inferior a $6,0 \mathrm{~m} \mathrm{e}$ estrato 2, de profundidade igual ou superior a $6,0 \mathrm{~m}$ ).

Foram obtidos, por amostra, dados da temperatura (através de termômetro de mercúrio, expressa em graus C), salinidade (através de refratômetro optico, expressa em \%o) e concentracão de oxigênio dissolvido (pelo método de Winkler modificado, expressa em mg/) para a água de superfície e de fundo; a profundidade foi obtida por ecobatímetro (expressa em metros).

Todos os exemplares de $S$. rastrifer capturados foram fixados em formalina a $10 \%$ e, em laboratório, os mesmos foram contados e medidos no comprimento total (mm); no mínimo, 40 exemplares de cada classe de comprimento foram medidos (mm) e pesados $(\mathrm{g})$ individualmente.

A estrutura da população foi analisada através das variaçōes temporais e espaciais das ocorrência e abundância (FOC e CPUE) ou distribuições das médias com $95 \%$ de intervalo de confiança, sendo os resultados expressos por mês, estrato de profundidade, setor e sub-setor (Figs 2.1, 2.2, 2.3 e 2.4).

Diferenças significativas entre os valores médios da abundância (CPUE) em função do tempo, espaço e variáveis hidrográficas foram verificadas pelo teste de Newman-Keuls $(P=0,05)$ (Sokal \& Rohlf, 1979).

A análise da estrutura da população foi, também, feita a partir da composição por comprimento, agrupando-se os indivíduos em classes de $5 \mathrm{~mm}$ de comprimento total (CT), ou através das médias de comprimento com intervalos de $95 \%$ de confiança, sendo os resultados expressos para todo o período de estudo, por mês, estrato de profundidade e sub-setor (Figs 3, 4.1 e 4.3).

Foram analisadas as variaçôes da abundância e do tamanho dos exemplares em relação à variaçōes de classes das temperatura $\left(2^{\circ} \mathrm{C}\right)$, salinidade $(2 \%$ ) e oxigênio dissolvido (1,5 mg/) (Figs 2.5 e 4.2).

O estudo do crescimento baseou-se na determinação da relação peso total/comprimento total, para todo o período e sem distinção de sexo; após inspeção gráfica e transformação logarítmica, os dados foram ajustados pelo método dos mínimos quadrados à expressão (Fig. 5.1):

$$
\mathbf{W t}=\mathbf{a} \mathbf{L t}^{\mathrm{b}},
$$

onde Wt é o peso total (g), Lt é o comprimento total (mm), aé o fator de condição alométrico e bé o coeficiente alométrico.

Através do programa ELEFAN (Electronic Length Frequency Analysis) (Brey \& Pauly, 1986), foi feita a análise da distribuição de frequêencia de comprimentos e reestruturação das mesmas . Para tanto, os comprimentos foram grupados em classes de $10 \mathrm{~mm}$ e introduzidos no programa; adotou-se valores de 1,0 e 0,6 para as constantes C (relacionada com a amplitude de oscilação de crescimento, a qual é dependente da variaçảo da temperatura da água) e WP (relacionada com a fração do mês em que o crescimento é menor, ou seja, nos meses mais frios), em função de ser julho o mês mais frio, e de que os melhores resultados, como um todo, foram obtidos com esses valores (Giannini, 1989).

Assim, o programa forneceu os valores de $\mathbf{L} \infty$ (comprimento total assintótico) e k (taxa de crescimento); a idade inicial (to) e a longevidade ( $t$ máx.) foram obtidas através das equaçōes empíricas de Pauly (1980):

$$
\begin{gathered}
\log (- \text { to })=-0,3922-0,2752 \log L_{\infty}-1,038 \log \mathrm{k} ; \\
\quad t \text { máx. }=\text { to }+2,9957 / \mathrm{k}
\end{gathered}
$$

A seguir foram calculados o comprimento e o peso totais médios por çasse etária, sem distinção de sexo, ajustando-se os resultados as curvas de crescimento em comprimento e em peso através da equação de von Bertalanffy (1938) (Figs 5.2 e 5.3):

$$
L t=L \infty\left[1-e^{-k(t-t o)}\right] ; W t=W \infty\left[1-e^{-k(t-t o)}\right]^{b},
$$

onde Lt é o comprimento total médio (mm) na idade

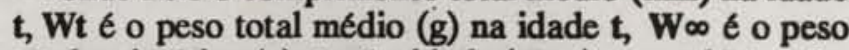
total assintótico (g), to é a idade (anos) na qual $\mathbf{L t}=0 \mathrm{e}$ t é a idade (anos).

Através de uma sub-rotina do programa ELEFAN 1, foram obtidos os comprimentos médios a cada mês, os quais foram usados na confecção da curva representativa do crescimento, obtida pela superposição dos histogramas de distribuição mensal de comprimentos e a melhor curva resultante da análise dos dados para uma única coorte (Fig. 6.1).

A determinação do padrão sazonal de recrutamento, da mortalidade e parâmetros correlatos, foi realizada através do programa ELEFAN (Brey \& Pauly, 1986); para tanto, após a obtenção dos valores de k, $L \infty, C$ e WP pelo programa ELEFAN 1, os mesmos foram introduzidos no programa ELEFAN 2 A.

O padrāo de recrutamento anual foi mostrado através de um gráfico no qual a porcentagem de recrutamento foi analisada em relaçäo a um período de um ano (Fig. 6.2).

Para a determinação da mortalidade e parâmetros correlatos, foi apresentada uma curva de captura (logarítmo natural da abundância nas várias classes de idade $x$ idade correspondente), na qual a parte descendente a direita da moda da curva pode ser assumida como a mortalidade da população (Fig. 6.3).

Foram calculados a seguir, o coeficiente de mortalidade natural (M) através da formula empírica de Pauly (1980), considerando-se o valor de $23,4^{\circ} \mathrm{C}$ como a temperatura média anual da água de fundo para a regiāo (Giannini, 1989): 

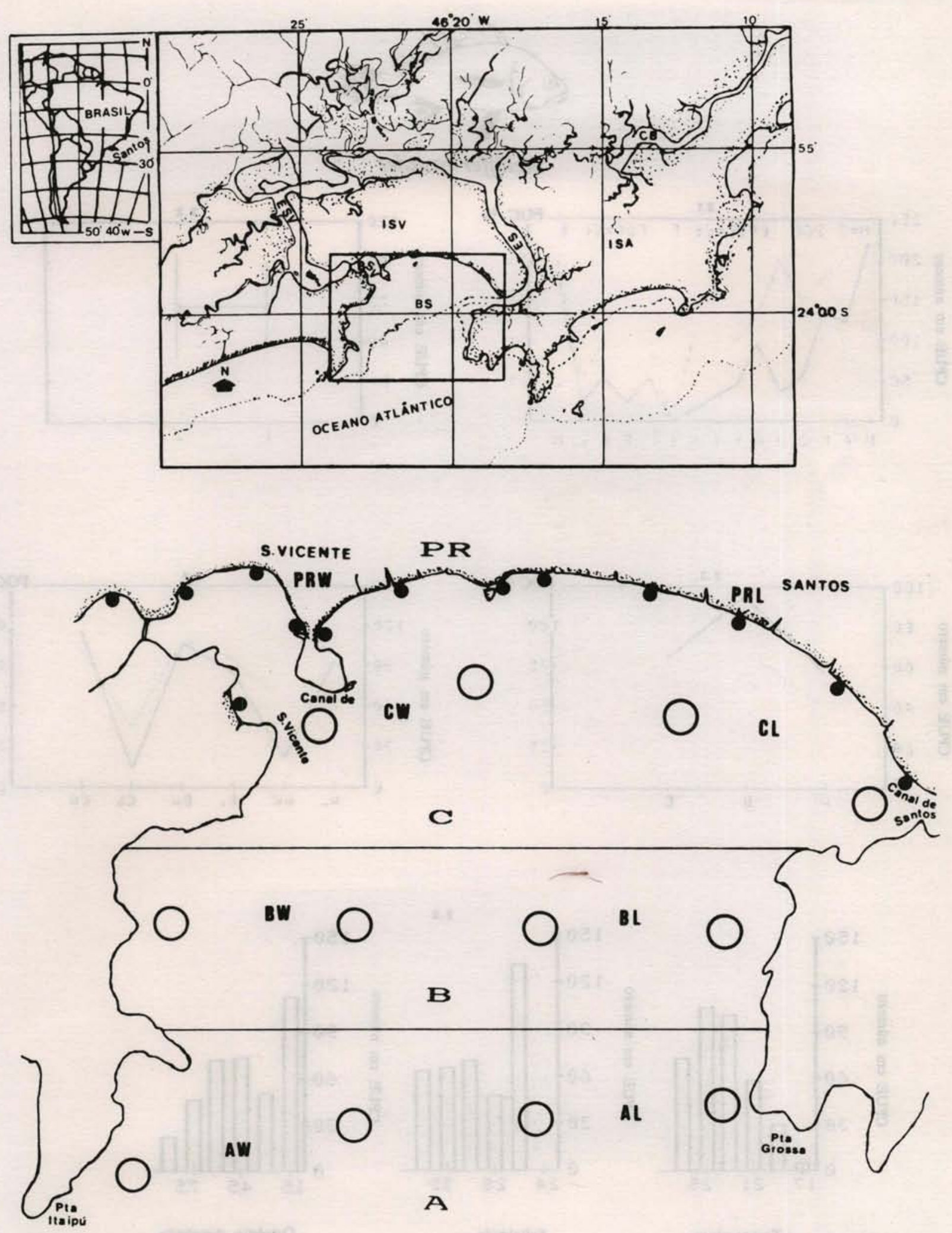

Fig. 1. Mapa da região de Santos e São Vicente, conforme carta da $\mathrm{DHN}$ no 1711, com as áreas de coleta assinaladas. As letras $A, B, C$ e PR, correspondem aos setores e as letras AL, AW, BL, BW, CL, CW, PRL e PRW aos sub-setores de amostragem. ISV (IIha de São Vicente); ISA (Illha de Santo Amaro); CB (Canal da Bertioga); ESV (Estuário de São Vicente); ES (Estuário de Santos); BSV (Baía de São Vicente); BS (Baía de Santos).

Arrastos de Fundo (O) Arrastos de Praia (O) 


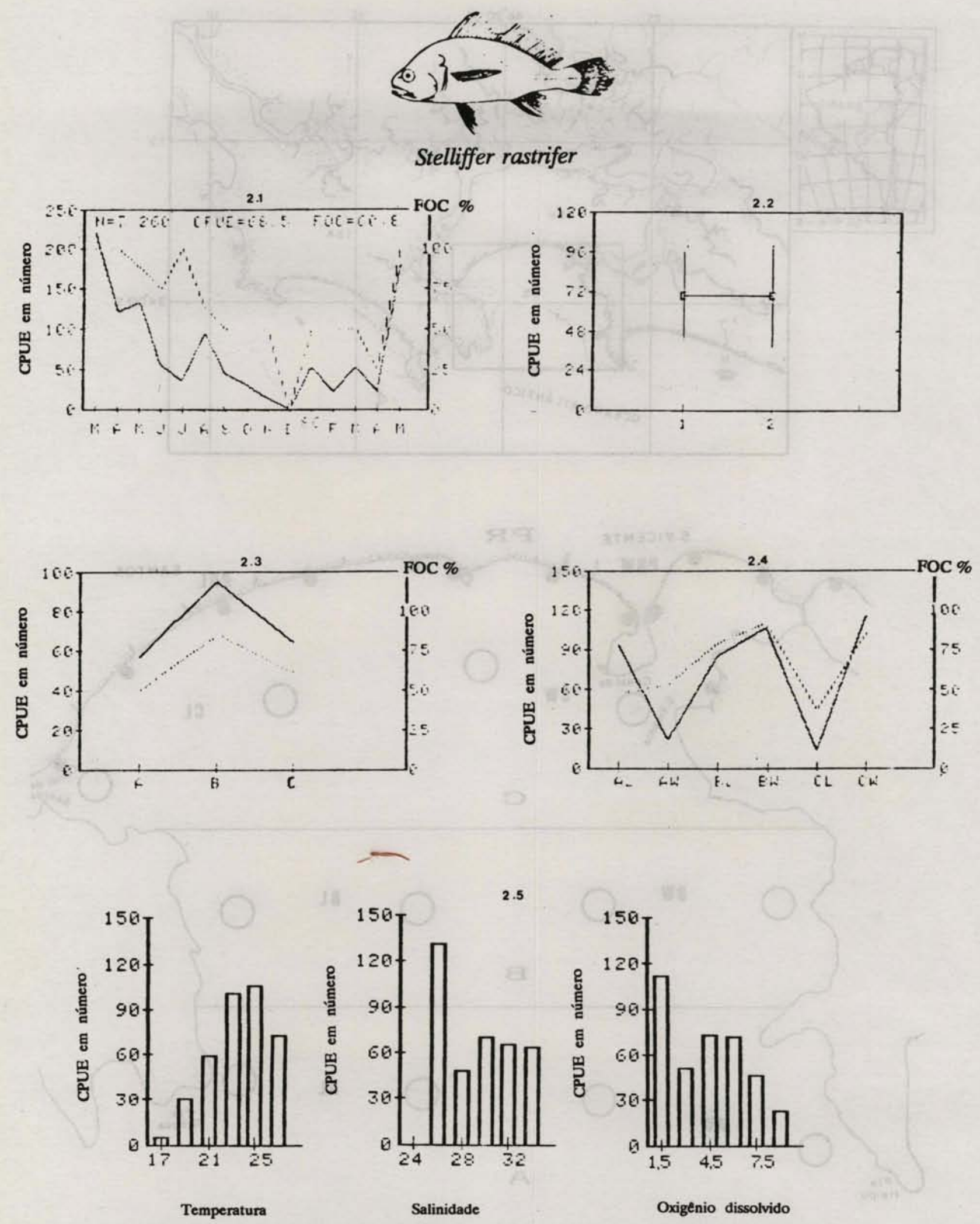

Fig. 2. Abundância relativa (CPUE) (linha contínua), expressa em número de exemplares e frequêência de ocorrência (FOC) (linha tracejada) das capturas médias mensais (2.1), por setor (2.3) e subsetor de coleta $(2.4)$ e por classes de temperatura $\left(2{ }^{\circ} \mathrm{C}\right)$, salinidade $(2 \% \circ)$ e oxigênio dissolvido $(1,5 \mathrm{mg} / \mathrm{l})(2.5)$, e média e intervalo de $95 \%$ de confiança para os estratos de profundidade (2.2), em amostras com arrastos de fundo. 

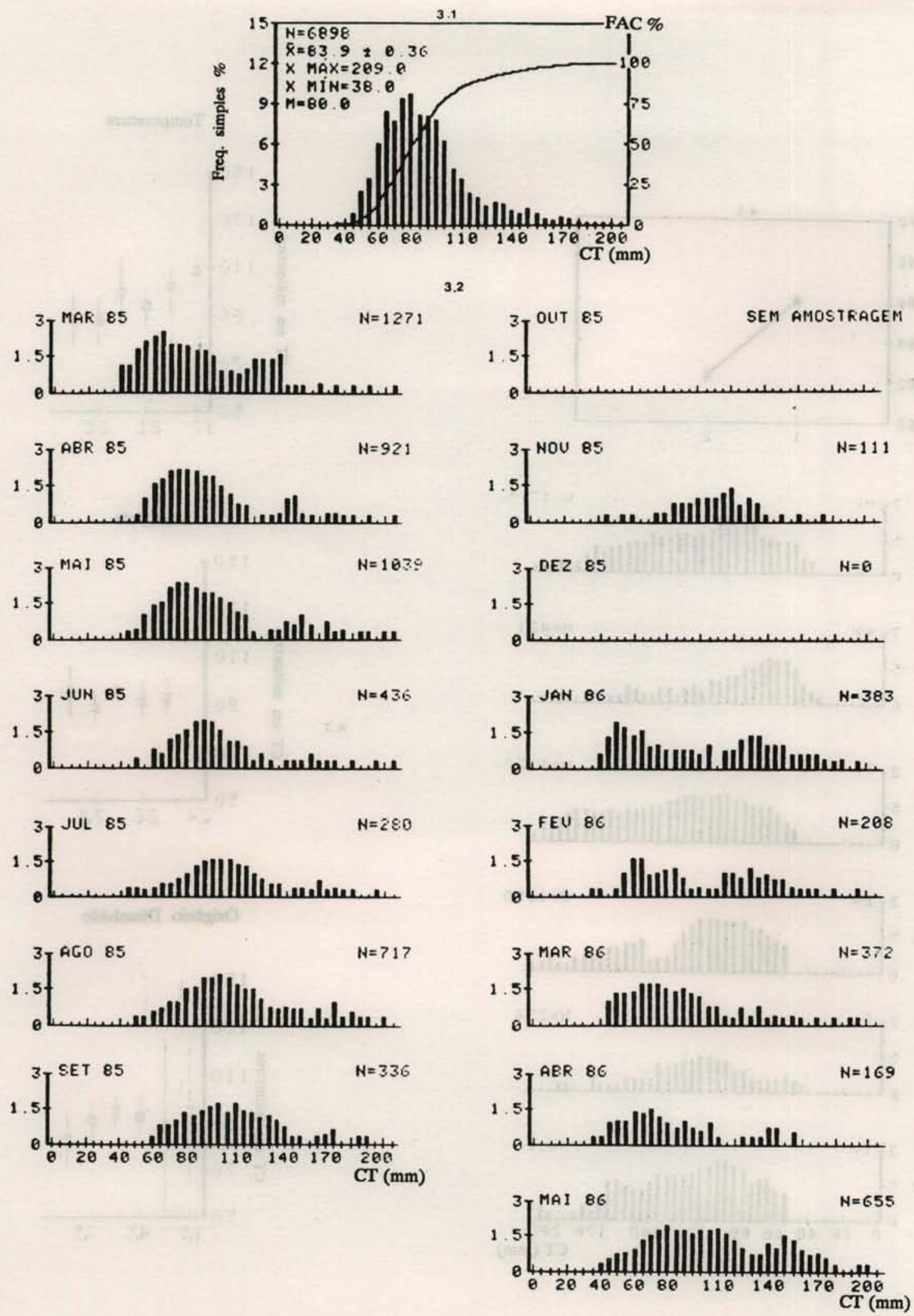

Fig. 3. Distribuição total de freqüência (FREQ SIMPLES \%) e freqüência acumulada (FAC \%) (3.1) e distribuição mensal de freqüência expressa em log $(N+1)(3.2)$, por classes de comprimento total CT $(5 \mathrm{~mm})$, em amostras com arrasto de fundo. 

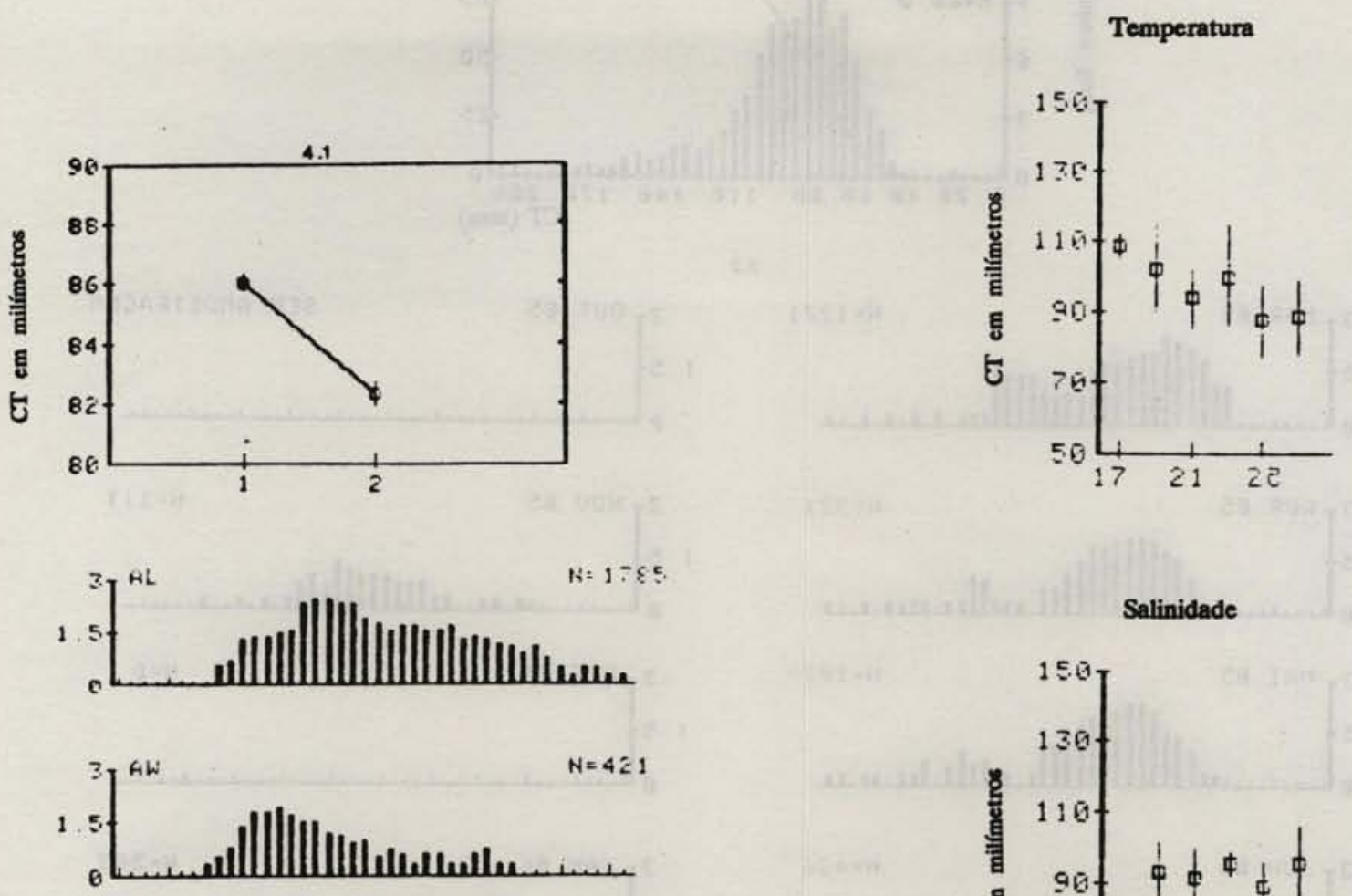

4.3
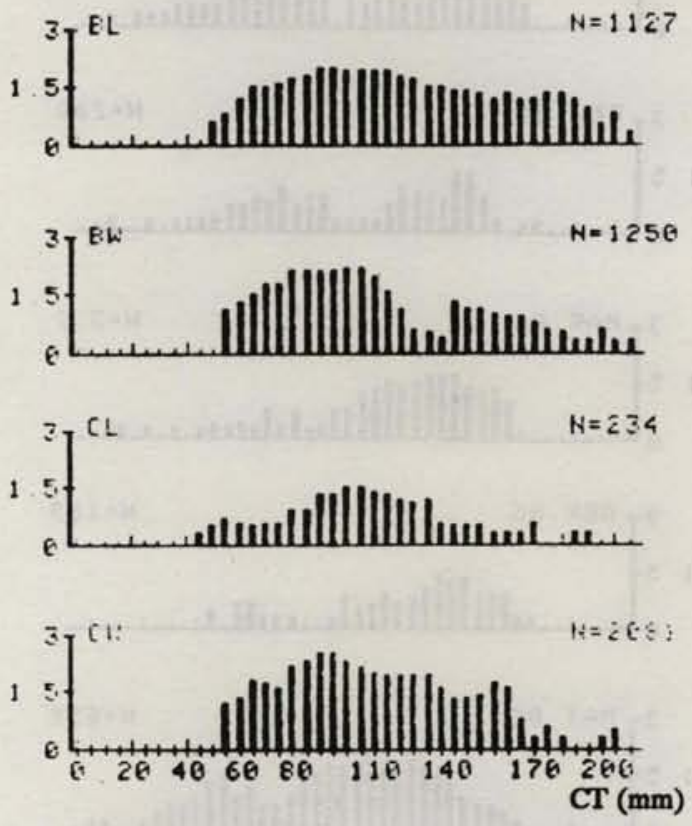

Salinidade

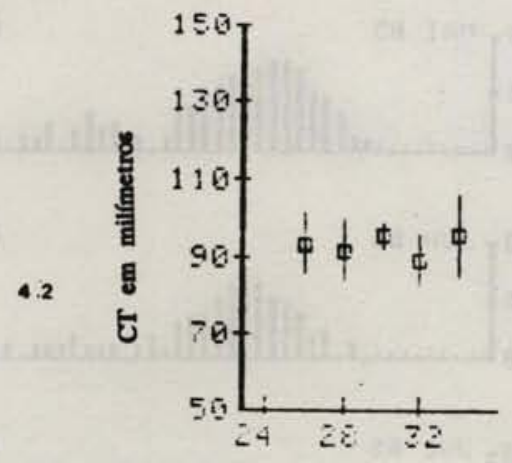

Oxigenio Dissolvido

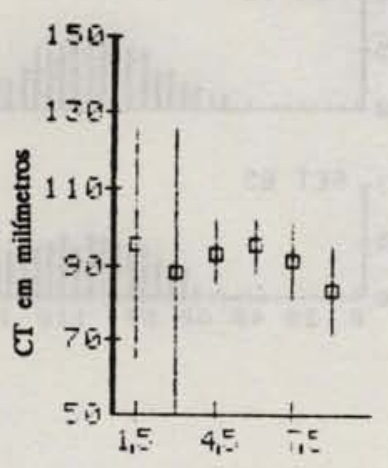

Fig. 4. Média e intervalo de $95 \%$ de conflança do comprimento total (em mm) para os estratos de profundidade $(4.1)$ e por classes de temperatura $\left(2{ }^{\circ} \mathrm{C}\right)$, salinidade $(2 \%$ ) e oxigênio dissolvido $(1,5 \mathrm{mg} / \mathrm{l})(4.2)$, e distribuição de frequeência por classes de comprimento total CT (5 $\mathrm{mm})$, expressa em log $(\mathrm{N}+1)$, por sub-setor de amostragem (4.3), em amostras com arrastos de fundo. 

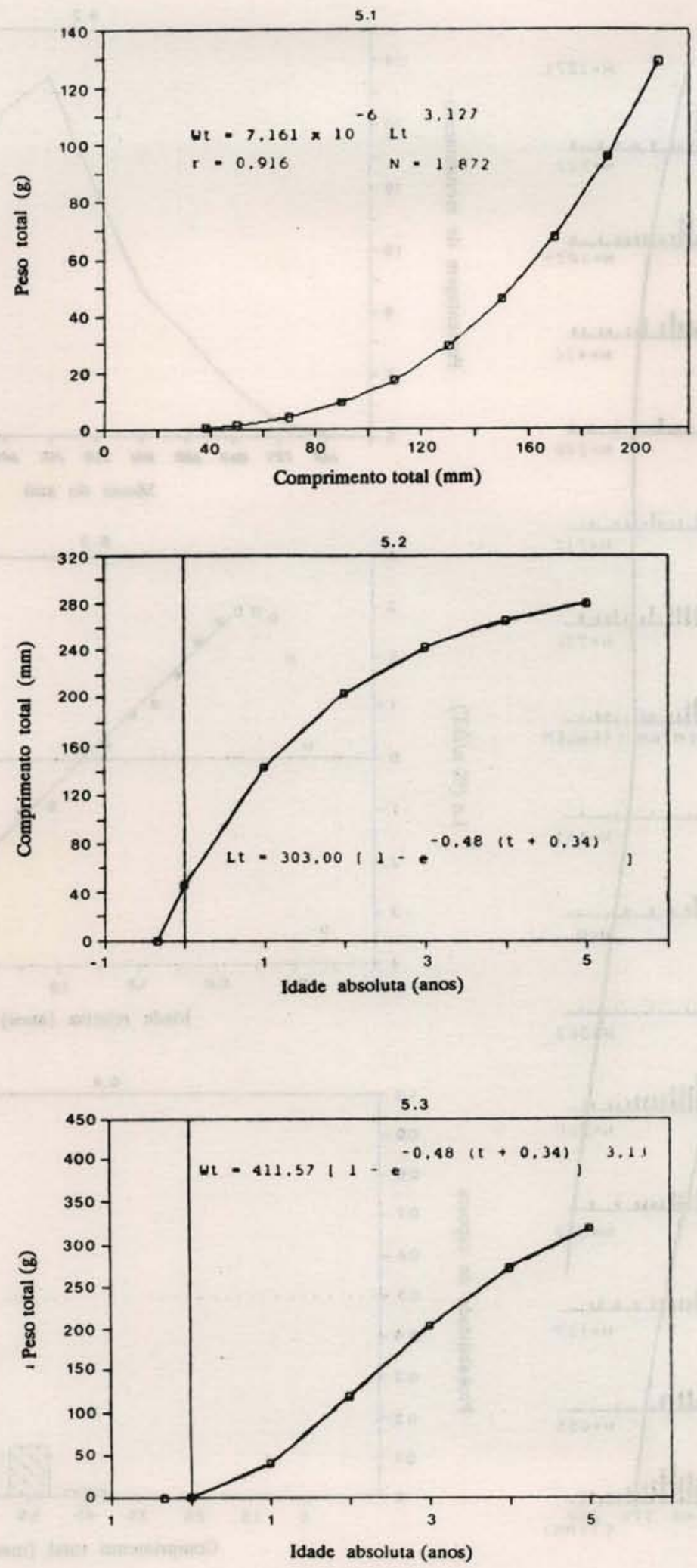

Fig. 5. Curvas representativas da relação peso total/comprimento total (5.1), e do crescimento em comprimento (5.2) e em peso (5.3) para sexos grupados, para todo o período estudado. 


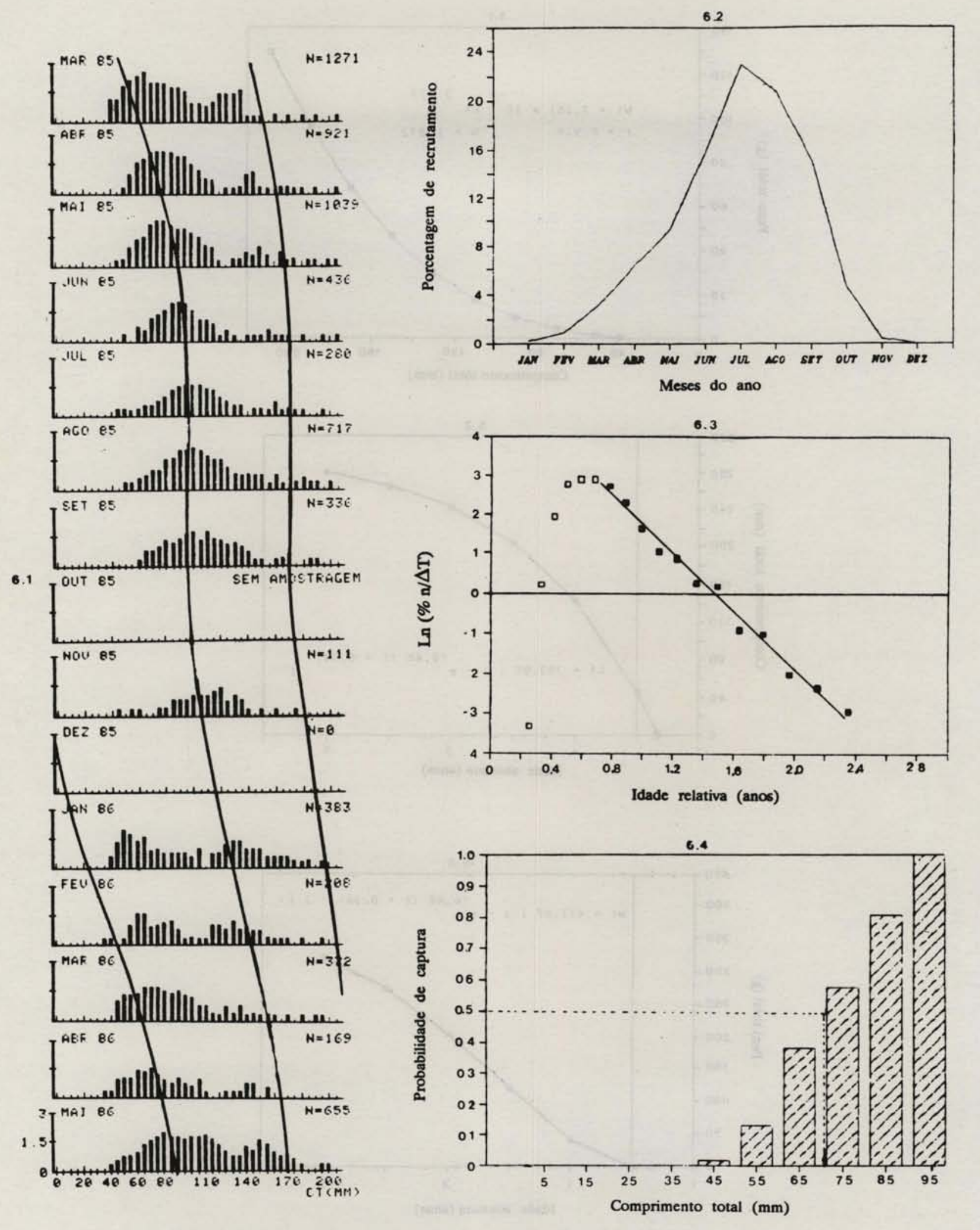

Fig. 6. Curvas representativas do crescimento (6.1), recrutamento (6.2 e 6.4) e mortalidade (6.3), para sexos grupados, para todo o período estudado. Parâmetros obtidos pelo programa ELEFAN: $L \infty=303,00 \mathrm{~mm} ; k=0,48 ; L c=70,80 \mathrm{~mm} ; Z=3,59 ; M=1,01$ e $E=0,72$ 


$$
\begin{aligned}
& \log M=-0,0066-0,279 \log L_{\infty}+0,6543 \log k+ \\
& 0,4634 \log \mathrm{T} \text {, }
\end{aligned}
$$

onde $\mathbf{T}$ é a temperatura média anual $\left({ }^{\circ} \mathrm{C}\right)$ da água na qual o estoque vive.

De posse dos valores dos coeficientes de mortalidade total (Z) e natural (M) e de correlação (r), o programa forneceu uma estimativa do tamanho médio de primeira captura $(\mathbf{L c})$, através dos valores computados da probabilidade de captura à cada classe de tamanho, os quais foram lançados em gráfico como histograma, onde o valor de $\mathbf{L} \boldsymbol{c}$ foi indicado pela linha tracejada (Fig. 6.4).

Os valores das taxas de mortalidade total (Z) e natural (M) assim obtidos, foram aplicados na equação de Gulland (1969), para a obtenção das taxas de mortalidade por pesca (F) e de explotação (E):

$$
\mathrm{F}=\mathrm{Z}-\mathrm{M} ; \mathrm{E}=\mathrm{F} / \mathrm{Z}
$$

onde $\mathbf{F}, \mathbf{Z}, \mathbf{M e} \mathbf{E}$ são as taxas de mortalidade por pesca, total, natural e de explotaçāo, respectivamente.

Uma descrição pormenorizada das etapas de processamento, equaçōes citadas, consideraçōes e premissas na utilização do programa ELEFAN foi descrita em Giannini (1989).

\section{Resultados e discussão}

\section{Estrutura da população em relação à abundância}

Stellifer rastrifer esteve praticamente restrita às amostras com arrasto de fundo. A frequêencia de ocorrência média (FOC) foi de $60,8 \%$, com um total de 7.260 exemplares capturados (CPUE de 68,5). Foi capturada durante quase todo o período amostrado (com excessão em dezembro), sendo significativamente ( $\mathrm{P}<$ 0,05) mais abundante em março de 1985 (Fig. 2.1).

A distribuição da espécie na área de estudo foi ampla, com capturas elevadas e muito semelhantes nos dois estratos de profundidade, ainda que com grandes flutuaçōes em torno da média (Fig. 2.2).

Altos valores da abundância numérica e da freqüência de ocorrência foram obtidos em todos os setores, com tendência para maiores abundâncias nos setores mediano e interno da baía (B e C) no lado oeste W, embora as capturas tenham sido semelhantes em toda a região; baixas capturas foram registradas nos sub-setores AW e CL, notadamente na Ponta de Itaipú e Canal de Santos. Os picos de abundância e de freqüência foram registrados para o setor B (CPUE de 93,0; FOC de $78,0 \%$, onde a espécie foi significativamente $(\mathrm{P}<0,05)$ mais abundante (Figs $2.3 \mathrm{e} 2.4$ ).

S. rastrifer apresentou maiores abundâncias em águas de temperatura entre $23 \mathrm{e} 25^{\circ} \mathrm{C}$ e de baixas concentraçôes de oxigênio dissolvido. Foi significativamente $(P<0,05)$ mais abundante na classe de salinidade de $26 \%$ (Fig. 2.5).

A homogeneidade para com os estratos de profundidade e áreas de amostragem (Figs 2.2, 2.3 e 2.4) indicaram a utilização uniforme de $S$. rastrifer na regiāo da Baía de Santos.

A ausência de capturas nas amostras com arrasto de praia indicou que os juvenis dessa espécie não utilizaram as zonas marginais rasas de praia, caracterizando um padrão de utilização mais restrito do ecossistema.

\section{Estrutura da população em relação ao comprimento}

S. rastrifer apresentou uma amplitude de comprimentos entre 38 e $209 \mathrm{~mm}$, com a moda principal nos $80 \mathrm{~mm}$ (CT); indivíduos maiores que $150 \mathrm{~mm}$ representaram menos de $10 \%$ das capturas (Fig. 3.1).

$O$ recrutamento dos juvenis (de comprimento entre 40 e $60 \mathrm{~mm}$ ), ocorreu a partir de janeiro, estendendo-se até maio; durante esse período a amplitude de comprimentos foi maior, sendo freqüente a captura de exemplares maiores que $150 \mathrm{~mm}$. De maio até setembro pode-se acompanhar, através do deslocamento das modas, o crescimento dos indivíduos na baía (Fig. 3.2).

Os menores exemplares foram capturados nas zonas mais profundas (estrato 2), embora a diferença da média dos comprimentos entre os dois estratos não fosse superior a $4 \mathrm{~mm}$ (Fig. 4.1).

Nos sub-setores AL e BL foram encontradas as maiores amplitudes de comprimentos e com distribuiçōes praticamente homogêneas; no sub-setor AW foram capturados os menores exemplares e a menor amplitude de comprimentos ( $40 \mathrm{a} 170 \mathrm{~mm}$ ), com a moda dos juvenis bem distinta. No sub-setor BW, a distribuição foi composta por duas modas distintas e no setor interno, em ambos os lados, a distribuição foi semelhante com modas um pouco superiores às dos demais sub-setores (Fig. 4.3).

$S$. rastrifer apresentou uma tendência dos menores exemplares ocuparem águas de temperaturas mais altas; em relação à salinidade e oxigênio, apresentou uma maior uniformidade de tamanhos ainda que com grandes flutuaçōes em torno da média, principalmente nas classes de concentração de oxigênio dissolvido entre 1,5 e $3,0 \mathrm{mg} / 1$ (Fig. 4.2).

Pela análise das variaçōes na abundância e no tamanho dos exemplares capturados durante o período de estudo, verificou-se que os jovens do ano foram capturados com os arrastos de fundo nas zonas mais profundas do setor externo, do verāo ao outono; posteriormente, os indivíduos passaram a ocupar os setores mais internos, de menores profundidades, de águas mais quentes e menos salinas, durante o inverno. Ao final deste, se concentraram nos setores mediano e interno, havendo um decréscimo de suas capturas na primavera, sendo que tal fato pode estar relacionado com um padrão de emigração em massa dos indivíduos pela proximidade da época de reprodução que, segundo Sinque (1977), ocorre no verão (Figs 2, 3 e 4).

\section{Crescimento}

Para a obtenção da relaçāo peso/comprimento, foram utilizados 1.872 exemplares, representativos de todas as classes de tamanho; a regressāo apresentou um 
coeficiente de correlação de Pearson (r) de 0,916, resultando na expressâo e curva da Figura 5.1.

$\mathrm{O}$ coeficiente de regressāo $\mathbf{b}$ foi muito semelhante ao obtido por Coelho et al. $(1985)(3,121)$, indicando que ambos os estudos abordaram a mesma populaçāo.

Pelo programa ELEFAN foram obtidos valores de 0,48 para $\mathbf{k}$ e de $303,00 \mathrm{~mm}$ para $\mathbf{L} \infty$; esse último, aplicado na equação representativa da relação peso/comprimento acima descrita, propiciou um valor de $411,57 \mathrm{~g}$ para $\mathrm{W} \infty$. Através das equaçōes de Pauly (1980), foram obtidos valores de - 0,34 anos para to e de 5,90 anos para t máx. As equaçōes e curvas representativas do crescimento em comprimento e em peso foram representadas nas Figuras 5.2 e 5.3.

Pelos resultados pode-se verificar que 0 valor obtido para $\mathbf{L} \infty$ foi bem superior ao sugerido em outros estudos (Chao, 1978; Menezes \& Figueiredo, 1980; Coelho et al., 1985), os quais estimaram um tamanho máximo de 200 $\mathrm{mm}$. Tal fato pode estar relacionado com a superestimação desse parâmetro pelo programa ELEFAN (Brey \& Pauly, 1986).

\section{Recrutamento e mortalidade}

A curva de crescimento do cangoá (Fig. 6.1), indicou a ocorrência de exemplares, na região, de classes etárias entre 0 e 2 anos. $O$ recrutamento pareceu ocorrer em um único pulso bem distinto (Fig. 6.2), representado pela entrada em massa de jovens nos meses de verāo-outono (Figs 2.1 e 3.2), resultado esse que difere do sugerido por Sinque (1977) que apontou duas desovas para a espécie (principal no verão); contudo, o reduzido número de exemplares amostrados pelo referido autor pode ter influenciado sua hipótese.

Os valores obtidos para Le e para o tamanho a partir do qual $100 \%$ dos indivíduos foram capturados (Fig. 6.4), foram compatíveis com as classes modais obtidas neste trabalho (Fig. 3.1) e no de Coelho et al. (1985) $(70 \mathrm{~mm})$. Os altos valores obtidos da mortalidade total $(\mathbf{Z})$ e da taxa de explotação (E) sugeriram que a espécie estaria submetida à uma alta pressão de pesca por esse tipo de aparelho.

Altos valores para esses parâmetros foram sugeridos por Coelho et al. (1985), em virtude da constatação de que a pesca estaria atuando sobre uma grande parcela de indivíduos imaturos.

Os resultados deste trabalho ratificaram essa hipótese, já que o comprimento de primeira maturação proposto pelos autores supracitados estaria situado na classe de $95 \mathrm{~mm}$, quando todos os indivíduos estariam recrutados à arte de pesca empregada (Fig. 6.4).

Esses altos valores são decorrentes não de uma pesca dirigida à espécie, mas possivelmente ao fato de que a mesma foi a mais abundante nas capturas (Giannini, 1989) e foi a principal espécie capturada na pesca dirigida ao camarāo sete-barbas Xyphopenaeus kroyeri na regiāo (Coelho et al., 1986; Paiva Filho \& Schmiegelow, 1986).

\section{Conclusões}

- Stellifer rastrifer apresentou capturas significativas somente nos arrastos de fundo, indicando uma utilização mais restrita do ecossistema na região.
- Sua distribuiçāo na Baía de Santos foi ampla, em águas rasas e profundas, sendo mais abundante e freqüente na porção intermediária.

- Os jovens do ano foram recrutados no verão-outono pelas zonas mais profundas, ocupando a seguir as áreas mais internas.

- Os altos valores das taxas de mortalidade total e de explotação sugeriram que a espécie estaria superexplorada pela arte de captura utilizada.

\section{Resumo}

Através de um programa de amostragem, com arrastos de praia e de fundo, no complexo baía-estuário de Santos e São Vicente, de março de 1985 a maio de 1986, foram capturados 7.260 exemplares de Stellifer rastrifer. Nesse trabalho foram analisados a ocorrência, a abundância, a distribuição de comprimentos, a influência de fatores ambientais, a relação peso/comprimento, o crescimento, o recrutamento e a mortalidade.

$S$. rastrifer foi capturada na regiāo durante todo o período e foi mais abundante e freqüente no outono, no lado oeste dos setores mediano e interno. Jovens do ano foram capturados primeiramente de janeiro a maio nas zonas mais profundas, migrando posteriormente para zonas mais rasas, de águas mais quentes e doces.

As baixas capturas encontradas na primavera podem estar relacionadas com uma emigraçāo em massa ao oceano, decorrentes de uma desova de verão.

$S$. rastrifer apresentou altos valores do coeficiente de crescimento e das taxas de mortalidade total e de explotação.

\section{Agradecimentos}

Os autores expressam seu agradecimento a Oscar Barbosa e Edgar Borges, da Base de Santos do Instituto Oceanográfico da Universidade de São Paulo e à tripulação do B/Pesq. "Veliger II", pela colaboração nas coletas.

Ao colega Francisco Borba Ribeiro Neto pelo apoio nos trabalhos de campo e de laboratório.

\section{Referências bibliográficas}

BERTALANFFY, L. von. 1938. A quantitative theory of organic growth (inquiries in growth laws II). Hum.Biol., 10:181-213.

BREY, T. \& PAULY, D. 1986. Electronic length frequency analysis: a revised and expanded user's guide to ELEFAN 0,1 , and 2. Ber. Inst. Meeresk. Christian-Albrechts Univ .,(149):1-76. 
CHAO, L.N. 1978. Sciaenidae. In: Fischer, W. ed. FAO species identification sheets for fishery purpose. Western Central Atlantic (fishing area 31). Rome, FAO. pag.var.

COELHO, J.A.P.; GRAÇA LOPES, R. da; RODRIGUES, E.S. \& PUZZI, A. 1985. Relação peso-comprimento e tamanho de início de primeira maturação gonadal para o Sciaenidae Stellifer rastrifer (Jordan, 1889), no litoral do Estado de São Paulo. Bolm Inst.Pesca, S Paulo, 12 (2):99-107.

; PUZZI, A.; GRAÇA LOPES, R. da; RODRIGUES, E.S. \& PIETRO JR., O. 1986. Análise da rejeição de peixes na pesca artesanal dirigida ao camaräo sete-barbas Xyphopenaeus kroyeri no litoral do Estado de São Paulo. Bolm Inst.Pesca, S Paulo, 13(2): 51-61.

GIANNINI, R. 1989. Distribuição temporal e espacial e aspectos bioecológicos da família Sciaenidae na Baía de Santos, SP, Brasil. Dissertaçāo de mestrado. Universidade de São Paulo, Instituto Oceanográfico. 203p.

GULLAND, J.A. 1969. Manual of methods for fishing stock assesment. Part I. Fish population analysis. Roma, FAO. 154 p.

MENEZES, N.A. \& FIGUEIREDO, J.L. 1980. Manual de peixes marinhos do sudeste do Brasil. IV. Teleostei (3). São Paulo, Museu de Zoologia da Universidade de São Paulo. 96p.
PAIVA FILHO, A.M.; GIANNINI, R.; RIBEIRO NETO, F.B. \& SCHMIEGELOW, J.M.M. 1987. Ictiofauna do complexo baía-estuário de Santos e Sāo Vicente, SP, Brasil. Relat. int. Inst. oceanogr. Univ. S Paulo, (17):1-10.

\& SCHMIEGELOW, J.M.M. 1986. Estudo sobre a ictiofauna acompanhante da pesca do camarảo sete-barbas, Xyphopenaeus kroyeri, nas proximidades da Baía de Santos, SP. Bolm Inst.oceanogr., S Paulo, 34 (único): 79-85.

PAULY, D. 1980. A selection of the simple methods for the assesment of tropical fish stocks. FAO Fish.Circ., (729):1-54.

RIBEIRO NETO, F.B. 1989. Estudo da comunidade de peixes da Baía de Santos, SP. Dissertaçāo de mestrado. Universidade de Sảo Paulo, Instituto Oceanográfico. 196p.

SINQUE, C. 1977. Distribuição do ictioplâncton na regiãolagunar de Cananéia e descrição das larvas da familia Sciaenidae (Teleostei). Tese de doutorado. Universidade de Sāo Paulo, Instituto de Biociências. 127p.

SOKAL, R.R. \& ROHLF, F.J. 1979. Biometria. Principios y métodos estadísticos en la investigación biológica. Madrid, H.Blumes Ediciones. 832p.

(Recebido em 08-06-89; aceito em 17-08-90) 